Journal Club

Editor's Note: These short, critical reviews of recent papers in the Journal, written exclusively by graduate students or postdoctoral fellows, are intended to summarize the important findings of the paper and provide additional insight and commentary. For more information on the format and purpose of the Journal Club, please see http://www.jneurosci.org/misc/ifa_features.shtml.

\title{
Distributed Representation of "What" and "Where" Information in the Parahippocampal Region
}

\author{
Antonio Fernández-Ruiz ${ }^{1,2}$ and Azahara Oliva ${ }^{1}$ \\ ${ }^{1}$ MTA-SZTE “Momentum” Oscillatory Neuronal Networks Research Group, Department of Physiology, University of Szeged, Szeged H-6720, Hungary, and \\ ${ }^{2}$ School of Physics, Complutense University, 28040 Madrid, Spain \\ Review of Keene et al.
}

A large body of evidence supports a key role for medial temporal lobe (MTL) structures in declarative memory. Initial theories assigned specific cognitive processes to particular subregions and suggested a hierarchical serial processing stream that converges in the hippocampus (Eichenbaum et al., 2007). Two main independent processing streams were proposed: the "what" stream, which encodes item features in the perirhinal cortex (PRC) and reaches the hippocampus through the lateral entorhinal cortex (LEC), and the "where" pathway, which encodes position and spatial context in the postrhinal cortex (POR) and reaches the hippocampus via the medial entorhinal cortex (MEC). In this framework, the role of the hippocampus is to integrate "what" and "where" information into conjunctive representations of "what happened where" and also "when," the basic components of episodic memory (Fig. 1).

This simple framework has been challenged by recent results showing mixed object and spatial coding in MEC and LEC (Knierim et al., 2013). Additionally, numer-

Received May 15, 2016; revised June 19, 2016; accepted June 23, 2016.

We thank Antal Berényi, György Buzsáki, and members of their laboratories for insightful comments and discussion. AF-R received funds from EMBO and Marie Curie Action (ALTF 147-2015).

The authors declare no competing financial interests.

Correspondence should be addressed to either Antonio Fernández-

Ruiz or Azahara Oliva, MTA-SZTE "Momentum" Oscillatory Neuronal Networks Research Group, Department of Physiology, University of Szeged, Szeged H-6720, Hungary. E-mail: antferrui@gmail.com or azaharaglez@gmail.com.

DOI:10.1523/JNEUROSCI.1581-16.2016

Copyright $\odot 2016$ the authors $\quad 0270-6474 / 16 / 368286-03 \$ 15.00 / 0$ ous anatomical interconnections exist at every level of the circuit and there are numerous bidirectional parallel pathways between hippocampal and entorhinal subregions. These findings suggest a more complex functional organization. Is the model of serial processing by independent functional modules still tenable or have enough evidence accumulated to support a theory in which "what" and "where" information is intermingled throughout distributed MTL networks?

A recent report (Keene et al., 2016) shines new light on this question. The authors recorded in MEC, LEC, and PRC regions as rats learned specific object-context associations that predicted reward. Animals learned to dig in two terra cotta pots with different texture and odor. In each of two contexts only one of the pots was always rewarded (different pot for each context), regardless of its position. Keene et al. (2016) found that all task dimensions (object position, object identity, context, and objectposition within context) were encoded in the three different areas, albeit to different degrees. This mixed representation was even present at the single-cell level. Cells that are traditionally considered purely spatialcoding cells (e.g., grid cells) showed strong selectivity for object identity in some cases, just as so-called "place cells" in the hippocampus encode objects under some circumstances. In tasks in which keeping track of elapsed time is important for reward, hippocampal and MEC cells show temporal coding as well (Kraus et al., 2013). These re- sults can be interpreted as an evidence of a more general phenomenon: associative cortical areas can flexibly support multiple representations according to behavioral demands. Keene et al. (2016) also raise a cautionary note: laboratory tasks are usually designed to test specific hypothesis about the function of a particular area and thus bias neuronal representations to those aspects that are task-relevant in a narrow context.

Keene et al. (2016) used population analysis to unveil ensemble representations of task dimensions. Although MEC, LEC, and PRC represented all relevant dimensions of the task at the population level, MEC ensembles displayed a stronger position coding than LEC and PRC ensembles, which preferentially encoded object identity. The authors also showed a qualitative difference in the nature of representational organization across the MTL. In the MEC, population representations of different positions within the same context were strongly segregated, whereas in LEC and PRC, the separation was stronger for object identity representations. These results illustrate that the organizing principle for information coding in MEC and PRC/LEC differ. MEC representations are organized primarily based on path integration mechanisms and self motion cues, whereas representations in LEC (and PRC) are organized based on local landmarks and individual item features (Knierim et al., 2013). These different computational mechanisms result in a stronger 


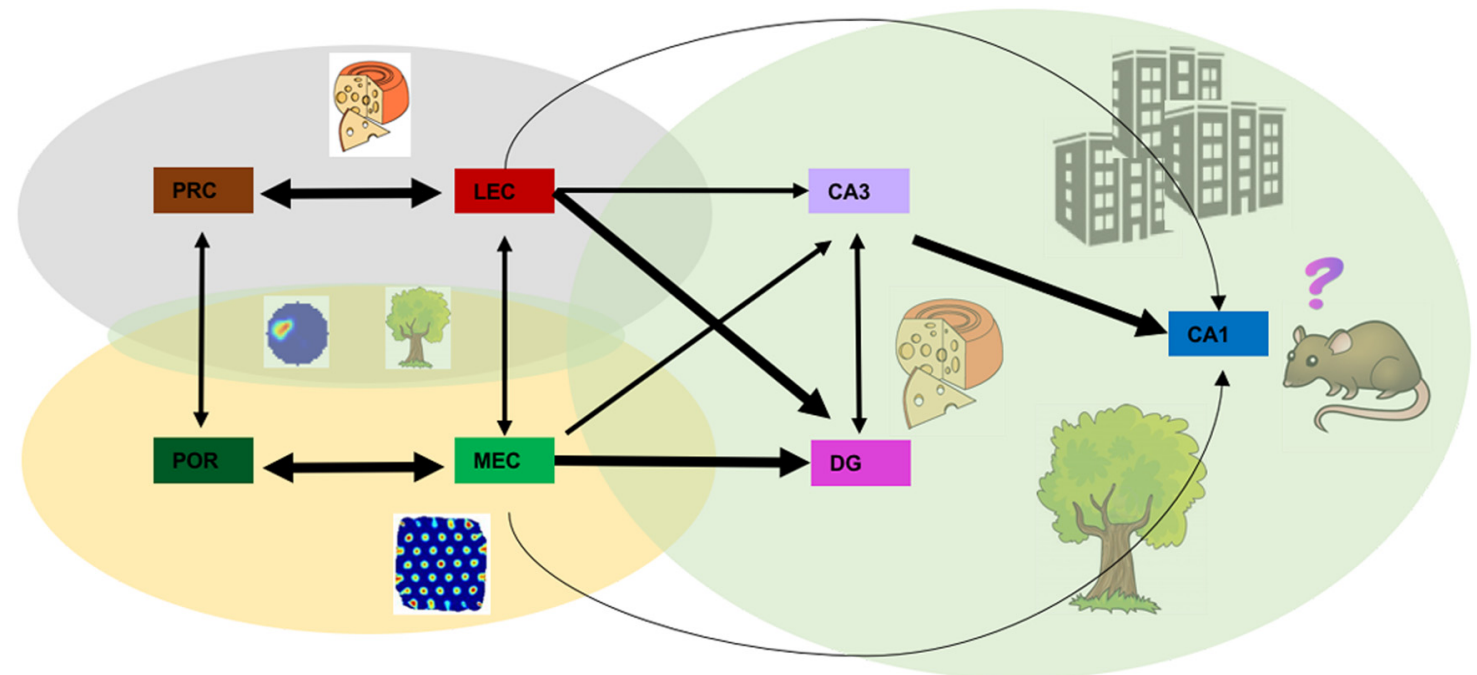

Figure 1. Schematic representation of information flow in the parahippocampal region. Black arrows indicate direction and strength of anatomical connections. Different processing streams are marked by different colored areas (gray for objects, yellow for position), with an overlapping surface (green) indicating conjunctive representations shown by Keene et al. (2016). In the hippocampus a complex multimodal pattern is formed.

position coding in MEC and stronger object identity in LEC and PRC.

The present study illustrates the usefulness of population analysis of neuronal representations (McKenzie et al., 2015). However, one caveat is that all analyses relied exclusively on firing rates. Although rate is a useful measure, much information is also stored in the temporal code of spikes. Investigating spike timing in relation to the concurrent theta and gamma rhythms would offer a deeper insight into the temporal organization of cells assemblies encoding different aspects of the task. For example, are MEC and LEC cells encoding position and object information firing during the same theta cycle in a specific sequence? Such "theta sequences" could offer a potential mechanism to integrate different information into a coherent representation. It has been shown that theta-gamma coupling in entorhino-hippocampal networks is essential for memory recall (Schomburg et al., 2014). Thus, it is likely that, in a similar way, local gamma oscillations synchronize the firing of cells encoding the same information while the global theta rhythm coordinate the activation of these cell assemblies across structures. Theta sequences and theta-gamma coupling could play a complementary role to the rate coding of object, position, and context shown by Keene et al. (2016).

If the representation of objects, context, and position is distributed across parahippocampal cortices, how is this information integrated into coherent episodic memories? The temporal analysis of Keene et al. (2016) suggest that position is first encoded in MEC and then sent to LEC, whereas the representation of objects follows the opposite path. Another possibility is that dentate granular cell ensembles that receive inputs from both LEC and MEC store these combined representations. In favor of this hypothesis, it has been shown that selective reactivation of granular cells active during a fear experience is sufficient to induce memory recall (Liu et al., 2012).

Nonetheless, the anatomical and experimental evidence suggest that the integration of different information modalities does not occur at only one level of the circuit. Both DG and CA3 integrate MEC and LEC inputs; however, they perform different computational operations on them. Others have found that the output of the DG rapidly decorrelates entorhinal inputs that reflect changes in the environment, whereas CA3 representations remain largely coherent, supporting a pattern separation function of DG and pattern completion function of CA3 (Neunuebel and Knierim, 2014). In contrast to CA3 and DG, MEC and LEC inputs do not overlap in CA1 (Amaral and Lavenex, 2007). MEC axons selectively innervate proximal CA1 (closer to CA3) and LEC axons distal CA1. This organization suggests that representations in proximal CA1 follow organizing principles similar to MEC (i.e., preferential spatial coding) and those in distal CA1 are similar to LEC/PRC. This hypothesis is supported by reports showing more precise spatial coding by proximal CA1 place cells (Henriksen et al., 2010) and stronger modulation by objects of distal CA1 place cells (Burke et al., 2011). However, CA1 cells also integrate conjunctive representations from CA3, so the information encoded in their firing patterns is likely more complex that those in the entorhinal cortex, as evidenced by the flexible expression of place and "time" cells in this region (Kraus et al., 2013). A possible reconciliation of these findings may be found in the temporal organization of the inputs arriving to CA1. Indeed, CA3 input dominates the first half of the theta cycle (descending phase) and entorhinal input the second (Schomburg et al., 2014), and the two halves of the theta cycle have been proposed to serve different functions, reflecting two coding modes of the CA1 network which serve respectively navigational and memory functions of the area (Sanders et al., 2015). According to this framework, during the descending phase of the theta cycle CA3 input drive CA1 place cells, representing current position of the animal, and update MEC grid cell representation. During the ascending theta phase, entorhinal input drive the firing of place cells representing possible future trajectories. This latter process could be useful to determine whether good or bad outcomes (i.e., finding reward) have been previously associated with potential upcoming positions.

The report by Keene et al. (2016) sets the stage for new work testing those hypotheses, although simultaneous recordings in all the implicated regions are required. Only such experiments can elucidate the cellular mechanisms of episodic memory formation and spatial coding in the temporal lobe.

\section{References}

Amaral D, Lavenex P (2007) Hippocampal neuroanatomy. In: The hippocampus book (Andersen P, Morris R, Amaral D, Bliss T, O’Keefe J, eds), pp 37-114. New York: Oxford UP. 
Burke SN, Maurer AP, Nematollahi S, Uprety AR, Wallace JL, Barnes CA (2011) The influence of objects on place field expression and size in distal hippocampal CA1. Hippocampus 21: 783-801. CrossRef Medline

Eichenbaum H, Yonelinas AP, Ranganath C (2007) The medial temporal lobe and recognition memory. Annu Rev Neurosci 30:123152. CrossRef Medline

Henriksen EJ, Colgin LL, Barnes CA, Witter MP, Moser MB, Moser EI (2010) Spatial representation along the proximodistal axis of CA1. Neuron 68:127-137. CrossRef Medline

Keene CS, Bladon J, McKenzie S, Liu CD, O'Keefe J, Eichenbaum H (2016) Complementary functional organization of neuronal activity patterns in the perirhinal, lateral entorhinal, and medial entorhinal cortices. J Neurosci 36: 3660-3675. CrossRef Medline

Knierim JJ, Neunuebel JP, Deshmukh SS (2013)
Functional correlates of the lateral and medial entorhinal cortex: objects, path integration and local-global reference frames. Philos Trans R Soc Lond B Biol Sci 369:20130369. CrossRef Medline

Kraus BJ, Robinson RJ 2nd, White JA, Eichenbaum H, Hasselmo ME (2013) Hippocampal "time cells": time versus path integration. Neuron 78:1090-1101. CrossRef Medline

Liu X, Ramirez S, Pang PT, Puryear CB, Govindarajan A, Deisseroth K, Tonegawa S (2012) Optogenetic stimulation of a hippocampal engram activates fear memory recall. Nature 484:381-385. CrossRef Medline

McKenzie S, Keene CS, Farovik A, Bladon J, Place R, Komorowski R, Eichenbaum H (2015) Representation of memories in the cortical-hippocampal system: results from the application of population similarity analyses. Neurobiol Learn Mem. Ad- vance online publication. Retrieved July 12, 2016. doi: 10.1016/j.nlm.2015.12.008 Medline

Neunuebel JP, Knierim JJ (2014) CA3 retrieves coherent representations from degraded input: direct evidence for CA3 pattern completion and dentate gyrus pattern separation. Neuron 81:416-427. CrossRef Medline

Sanders H, Rennó-Costa C, Idiart M, Lisman J (2015) Grid cells and place cells: an integrated view of their navigational and memory function. Trends Neurosci 38:763-775. CrossRef Medline

Schomburg EW, Fernández-Ruiz A, Mizuseki K, Berényi A, Anastassiou CA, Koch C, Buzsáki G (2014) Theta phase segregation of input-specific gamma patterns in entorhinal-hippocampal networks. Neuron 84:470-485. CrossRef Medline 